\title{
EXAME CLÍNICO OBJETIVO ESTRUTURADO: \\ REFLEXÕES SOB UM OLHAR DA ENFERMAGEM*
}

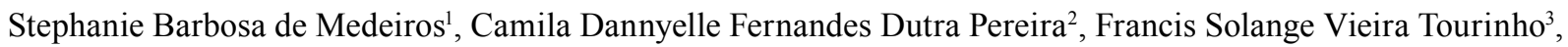
Liva Gurgel Guerra Fernandes ${ }^{2}$, Viviane Euzébia Pereira Santos ${ }^{3}$

RESUMO: Este artigo de reflexão objetiva discorrer sobre o Exame Clínico Objetivo Estruturado e refletir sobre o seu uso na formação dos alunos de enfermagem. Trata-se de exame criteriosamente estruturado que permite melhorar a validade e confiabilidade da avaliação de aspectos das competências clínicas dos alunos. Na enfermagem, o Exame Clínico Objetivo Estruturado é introduzido como ferramenta de avaliação na graduação, contribuindo para melhorar o processo de formação e, apesar da crescente utilização, são escassos os estudos realizados por enfermeiros, constituindo um desafio para a busca de maior conhecimento científico por meio de pesquisas. DESCRITORES: Enfermagem; Educação em enfermagem; Pesquisa em enfermagem.

\section{EXAMEN CLÍNICO OBJETIVO ESTRUCTURADO: REFLEXIONES BAJO LA VISIÓN DE LA ENFERMERÍA}

RESUMEN: Este artículo de reflexión tiene el objetivo de discutir sobre el Examen Clínico Objetivo Estructurado y reflexionar acerca de su uso en la formación de los alumnos de enfermería. Es un examen estructurado con criterio que permite mejorar la validad y confiabilidad de la evaluación de aspectos de las competencias clínicas de los alumnos. En la enfermería, el Examen Clínico Objetivo Estructurado es introducido como herramienta de evaluación en la graduación, contribuyendo para mejorar el proceso de formación y, a pesar de la creciente utilización, son escasos los estudios realizados por enfermeros, constituyendo un desafío para la búsqueda de más conocimiento científico por medio de investigaciones.

DESCRIPTORES: Enfermería; Educación en enfermería; Investigación en enfermería.

\section{OBJECTIVE STRUCTURED CLINICAL EXAMINATIONS: REFLECTIONS FROM A NURSING PERSPECTIVE*}

\begin{abstract}
This reflexive article aims to discuss the objective structured clinical examination and reflect on its use in the training of student nurses. It is a carefully structured examination which allows the improvement of the validity and reliability of the evaluation of aspects of the students' clinical competencies. In nursing, the Objective Structured Clinical Examination has been introduced as an evaluative tool on undergraduate courses, contributing to improve the training process; however, in spite of its growing use, few studies have been undertaken by nurses, constituting a challenge for the search for greater scientific knowledge through research.
\end{abstract}

KEY WORDS: Nursing; Education in nursing; Research in nursing.

*Manuscrito vencedor do prêmio Rosi Maria Koch no $64^{\circ}$ Congresso Brasileiro de Enfermagem, 2012.

${ }^{1}$ Enfermeira. Mestranda em Enfermagem. Bolsista CNPq. Universidade Federal do Rio Grande do Norte. Natal-RN-Brasil ${ }^{2}$ Enfermeira. Mestranda em Enfermagem. Universidade Federal do Rio Grande do Norte. Natal-RN-Brasil

${ }^{3}$ Enfermeira. Doutora em Enfermagem. Professora da Universidade Federal do Rio Grande do Norte. Natal-RN-Brasil 


\section{INTRODUÇÃO}

Ao longo dos últimos 50 anos os métodos de avaliação dos alunos têm apresentado mudanças, tornando-se cada vez mais complexos. Nas três décadas de 19651995 registrou-se crescimento nos esforços para aquisição de inovações na educação, passando-se de um padrão de papel e caneta para formas mais evoluídas de avaliar os conhecimentos ${ }^{(1)}$.

No campo da saúde, a avaliação das competências muitas vezes representa um desafio para os programas de formação, seja na graduação como na pós-graduação. Existem modelos tradicionais, como exames orais e perguntas de múltipla escolha, que se concentram na avaliação de habilidades clínicas; e modelos diferenciados, podendo neste caso, citar o Exame Clínico Objetivo Estruturado, do inglês Objective Structured Clinical Examination (OSCE) ${ }^{(2)}$.

Criado desde a década de 1970 por Harden, o OSCE se tornou um método avaliativo popular, principalmente nos Estados Unidos onde foi incorporado ao exame de licenciamento de medicina do país. No entanto, ainda existem repercussões sobre sua superioridade em comparação aos métodos tradicionais de avaliação clínica ${ }^{(1)}$. O referido Exame Clínico consiste em um exame de avaliação de habilidades clínicas e competências baseados no desempenho de uma forma confiável e válida, sendo muito utilizado, originalmente, na educação médica ${ }^{(2)}$, além de outros contextos da saúde, como na enfermagem e odontologia ${ }^{(3)}$.

Refere-se a um exame organizado em várias estações as quais os estudantes rodam e são avaliados em tarefas específicas (anamnese, exame físico, comunicação com o paciente, entre outras) ${ }^{(3)}$ por um ou dois examinadores, que pontuam o desempenho em uma folha de marcação/tempo previamente estruturados ${ }^{(4-7)}$. Apesar de ainda ser diferente da realidade vivenciada na prática clínica, por avaliar de forma fragmentada, o OSCE foi pensado para aproximar-se desta realidade ${ }^{(8)}$.

As etapas do OSCE avaliam, individualmente, as competências no que diz respeito à comunicação, bioética, aspectos pedagógicos e avaliação $\mathrm{crítica}^{(2)}$. Assim, o OSCE é fundamental para a identificação de lacunas de perspicácia clínica em alunos, e eficaz para corrigir as deficiências que necessitam de tempo dos professores. Além disso, o exame proporciona excelente oportunidade para os alunos refletirem sobre $o$ seu desempenho e capacidades/habilidades clínicas ${ }^{(9)}$.

Por tratar-se de um método recentemente utilizado pela enfermagem, somado a lacuna existente nos estudos acerca do OSCE por esta área do campo da saúde, decidiu-se por realizar este estudo que objetiva discorrer sobre o Exame Clínico Objetivo Estruturado e refletir sobre seu uso na formação dos alunos de enfermagem.

\section{EXAME CLÍNICO OBJETIVO ESTRUTURADO}

O OSCE é um exame criteriosamente estruturado, o que determina a diminuição da subjetividade por parte dos docentes examinadores ${ }^{(2)}$. Possui a capacidade de melhorar a validade e confiabilidade das avaliações de muitos aspectos das competências clínicas, podendo ser realizado conjuntamente a outro tipo de método de avaliação para maior confiabilidade, além de ser embasado por grande gama de evidências científicas ${ }^{(4)}$.

A padronização deste método avaliativo é obtida utilizando-se: 1) cenários simulados - próximos da realidade da prática clínica - semelhantes para todos os candidatos; 2) pacientes padrões, treinados para retratar de forma eficaz situações clínicas, garantindo que todos os alunos enfrentem situações similares ${ }^{(6)}$; $3)$ examinadores qualificados aos quais podem ser oferecidos treinamentos apontando os princípios do funcionamento do exame e a compreensão do papel que possuem frente a esse ${ }^{(6)}$; 4) listas de verificação cuidadosamente elaboradas/revisadas para identificar elementos específicos de conhecimentos e habilidades do exame, permitindo reflexo do verdadeiro desempenho do examinado ${ }^{(4)}$; 5) pontuação, que quantifica $o$ desempenho do aluno nas habilidades avaliadas ${ }^{(2,10)}$.

\section{Passos para o desenvolvimento do Exame Clínico Objetivo Estruturado}

Para a criação de um OSCE é necessário, primeiramente, estabelecer o que deve ser avaliado, posto que este tipo de exame não é apropriado para avaliar todos os aspectos da competência clínica. As atividades escolhidas devem estar em consonância com o nível de aprendizagem dos discentes e os objetivos do curso. Sua viabilidade também deve ser levada em consideração, sendo indispensável o uso de um modelo para planejar um exame ${ }^{(6)}$.

Sendo assim, alguns pontos são referidos como de grande utilidade para o funcionamento das estações, tais como: oferecer instruções para alunos e examinadores (informar ao discente claramente a tarefa que deve ser executada e aos examinadores a sua função e como conduzir a estação); listar os materiais neces- 
sários; estabelecer o cenário simulado e o cronograma (aspectos importantes e tempo de cada estação); decidir a necessidade de um paciente padrão ou real e as características de cada indivíduo, como sexo e idade ${ }^{(6)}$.

Outros aspectos também apontados na literatura para o bom funcionamento do OSCE são ${ }^{(6)}$ :

- Anteriormente ao OSCE: as estações podem estar em uma única sala compartimentalizada ou serem realizadas em salas separadas; dependendo da quantidade de estações e de candidatos, mais de um circuito pode ser conduzido simultaneamente. $\mathrm{O}$ recrutamento de examinadores e dos pacientes padrões deve ser feito por meio de um aviso prévio e as estações devem estar numeradas evitando confusão com materiais e pessoas envolvidas. Todo o material necessário por estação deve ser listado e verificado previamente ao dia da aplicação do exame.

- No dia da aplicação do OSCE: a sinalização deve estar bem clara, destacando as salas, permitidas para os alunos, pacientes e aquelas onde esse será realizado. O tempo dos discentes em cada estação deve ser controlado, desde que seja garantido que alunos e examinadores possam escutar a sonorização utilizada nitidamente; são necessários ajudantes para nortear candidatos, examinadores e pacientes.

- Depois do OSCE: as folhas com as pontuações devem ser organizadas minuciosamente.

\section{Limitações e Benefícios do Exame Clínico Objetivo Estruturado}

Embora haja grande aceitação deste exame, algumas limitações são referidas na literatura, tais como: altos custos para implementação do OSCE (instalações, finanças, pessoal) quando comparado a outros métodos de avaliação; tempo destinado para treinamento dos examinadores e pacientes padrões; manutenção do espaço em que ocorrem os exames; além da necessidade de maiores evidências que comprovem a validade de sua utilização ${ }^{(1-2)}$.

Não obstante as barreiras potenciais acerca do OSCE, relatos têm destacado diversos benefícios oriundos deste exame. Nesses estudos pôde-se comprovar que o desempenho dos avaliados melhorou, permitiu a padronização de pequenos grupos de ensino, o feedback para os professores que receberam informações sobre em que aspectos a aprendizagem das habilidades deveria melhorar, além de reduzir a ansiedade e aumentar da confiança diante dos rodízios da prática. Assim, mediante o uso do OSCE, obtêm-se resultados altamente válidos e importantes para tomada de decisões ${ }^{(1)}$.

\section{Exame Clínico Objetivo Estruturado na formação em Enfermagem}

A formação acadêmica do enfermeiro tem sido objeto de reflexão e estudo ao longo dos anos. As novas diretrizes curriculares para a Graduação em Enfermagem têm direcionado as mudanças na formação deste profissional, exigindo formação mais crítica, reflexiva flexível e versátil $^{(11)}$. Nesse sentido, o OSCE vem sendo introduzido com ferramenta de avaliação dos alunos em enfermagem.

Dentro deste contexto, foi desenvolvido, após aprovação do Comitê de Ética e Pesquisa/UFRN (parecer n. 147/2012), um projeto de pesquisa intitulado "Segurança do paciente: conhecer para promover a segurança no cuidado de enfermagem", o qual apresentava, dentre suas etapas metodológicas, a realização do OSCE no Laboratório de Habilidades do Centro Ciências da Saúde da referida instituição de ensino; este projeto está em implantação na disciplina de Atenção Integral II, na área de alta complexidade.

O OSCE desenvolvido foi esquematizado previamente em quatro estações baseadas em algumas das metas internacionais para a segurança do paciente, a saber: 1) promover adequada higienização das mãos; 2) melhorar a segurança na medicação; 3) identificar os pacientes corretamente; 4) promover comunicação efetiva (passagem de plantão) ${ }^{(12)}$.

Diante da realização deste exame, os estudantes avaliados tiveram a oportunidade de vivenciar situações semelhantes às encontradas na prática clínica, sendo possível que estes refletissem acerca das dificuldades resultantes das temáticas abordadas no OSCE, das responsabilidades resultantes destas atribuições, da comunicação necessária, tanto com o paciente como com a equipe de trabalho, contribuindo, assim, para a formação de um profissional capacitado para prestar assistência eficaz e de qualidade.

No que concerne aos docentes, estes puderam visualizar mais facilmente os alunos que apresentaram dificuldades, podendo também intervir de forma mais eficaz para melhor processo de aprendizagem. Tudo isso constitui evidências para a relevância da utilização do OSCE na enfermagem, o que exige dos envolvidos no processo de ensino aprendizagem a reflexão em torno do emprego deste método de avaliação.

\section{CONSIDERAÇÕES FINAIS}

A partir do exposto, pode-se aferir que o OSCE bem sucedido resulta de planejamento e coordenação bem estruturados, utilizando diversos recursos e crite 
riosos dados de avaliação. Em meio a toda a expansão do uso deste exame, pelos vários cursos da área da saúde, é fundamental atentar para a minimização da ocorrência dos erros.

No cenário da enfermagem, o OSCE vem conquistando espaço como instrumento avaliativo no processo de ensino-aprendizagem. No entanto, apesar da crescente utilização deste método, são escassos os estudos realizados por enfermeiros acerca da temática. Assim, faz-se necessário a reflexão por parte destes profissionais para a busca de maior conhecimento científico através do desenvolvimento de mais pesquisas sobre o OSCE, sua confiabilidade e validade, como também os benefícios resultantes para os estudantes, os docentes e a prática clínica.

\section{REFERÊNCIAS}

1. Turner JL, Dankoski ME. Objective Structured Clinical Exams: A Critical Review. Fam Med. [Internet] 2008;40(8):574-8 [acessoem 10ago 2012].Disponível:http:// www.stfm.org/fmhub/fm2008/September/John574.pdf

2. Jefferies A, Simmons B, Tabak D, Mcilroy JH, Lee $\mathrm{KS}$, Roukema $\mathrm{H}$, et al. Using an objective structured clinical examination (OSCE) to assess multiple physician competencies in postgraduate training. Med Teach. [Internet] 2007;29(2-3) [acesso em 12 ago 2012]. Disponível: http://informahealthcare.com/doi/ abs/10.1080/01421590701302290

3. Dennehy PC, Susarla SM, Karimbux NY. Relationship Between Dental Students' Performance on Standardized Multiple-Choice Examinations and OSCEs. Journal of J Dent Educ. [Internet] 2008;72(5) [acesso em 10 ago 2012]. Disponível: http://www.jdentaled.org/ content/72/5/585.full.pdf + html

4. Newble D. Techniques for measuring clinical competence: objective structured clinical examinations. Med Educ. [Internet] 2004;38(2) [acesso em 10 ago 2012]. Disponível: http://onlinelibrary.wiley.com/ doi/10.1111/j.1365-2923.2004.01755.x/pdf

5. Ronald ME. Assessment in Medical Education. N Engl J Med. [Internet] 2007;356(4) [acesso em 11 ago 2012]. Disponível: http://www.nejm.org/doi/full/10.1056/ NEJMra054784

6. Boursicot K, Roberts T. How to set up an OSCE. Clin. teach. [Internet] 2005;2(1) [acesso em 11 ago 2012]. Disponível: http://onlinelibrary.wiley.com/doi/10.1111/ j.1743-498X.2005.00053.x/pdf
7. Patrício M, Julião M, Fareleira F, Young M, Norman G, Carneiro AV. A comprehensive checklist for reporting the use of OSCEs. Med Teach. [Internet] 2009;31(2) [acesso em 11 ago 2012]. Disponível: http://informahealthcare. com/doi/abs/10.1080/01421590802578277

8. Van der Vleuten CPM, Schuwirth LWT, Scheele F, Driessen EW, Hodges B. The assessment of professional competence: building blocks for theory development. Best Pract Res Clin Obstet Gynaecol. [Internet] 2010;24(6) [acesso em 11 ago 2012]. Disponível: http://www.sciencedirect.com/science/article/pii/ S1521693410000519\#

9. White CB, Ross PT, Gruppen LD. Remediating Students' Failed OSCE Performances at One School: The Effects of Self-Assessment, Reflection, and Feedback. Acad Med. [Internet] 2009;84(5) [acesso em 10 ago 2012]. Disponível: http://www.ncbi.nlm.nih.gov/ pubmed/19704203

10. Payne NJ, Bradley EB, Heald EB, Maughan KL, Michaelsen VE, Wang XQ, et al. Sharpening the Eye of the OSCE with Critical Action Analysis. Acad Med. [Internet] 2008;83(10) [acesso em 11 ago 2012]. Disponível: http://www.ncbi.nlm.nih.gov/ pubmed/18820517

11. Martinéli DD, Moura CR, Cesarino CB, Beccaria LM, Pinto MH, Paschoal VDA. Avaliação do currículo da graduação em enfermagem por egressos. Cogitare enferm. 2011;16(3):524-9.

12. Portal da Enfermagem. [Internet] 2010 [acesso em 12 ago 2012]. Disponível: http://www.portaldaenfermagem. com.br/entrevistas_read.asp?id $=42$ 